levels of trust and reciprocity were related to non-smoking and adequate duration of sleep, and high levels of social support to adequate duration of sleep and daily use of vegetables. These associations persisted after controlling for age, gender, education and living arrangements. According to our findings, structural social capital seems to be associated with health behaviour more strongly than cognitive social capital.

Conclusion Irrespective of their social status, people with higher levels of social capital-especially in social participation and networks-engage in healthier behaviour. When trying to reduce health inequalities, one strategy could be to promote social participation especially among people in danger of social exclusion.

\section{P32 USING EVIDENCE TO PRIORITISE AREAS FOR PUBLIC HEALTH ACTIONS FOR TACKLING CHILDHOOD OVERWEIGHT}

\section{doi:10.1136/jech.2010.120477.32}

${ }^{1} \mathrm{G}$ McNeill, ${ }^{1} \mathrm{G}$ Osei-Assibey, ${ }^{1} \mathrm{~S}$ Dick, ${ }^{1} \mathrm{~J}$ Macdiarmid, ${ }^{1,2} \mathrm{~S}$ Semple, ${ }^{3} \mathrm{~J}$ Reilly, ${ }^{4} \mathrm{~A}$ Ellaway, ${ }^{2} \mathrm{H}$ Cowie. ${ }^{1}$ Population Health Section, University of Aberdeen, Aberdeen, UK; ${ }^{2}$ Division of Developmental Medicine, University of Glasgow, Glasgow, UK; ${ }^{3} \mathrm{MRC}$ Social and Public Health Sciences Unit, Glasgow, UK; ${ }^{4}$ Institute of Occupational Medicine, Edinburgh, UK

Objective To use evidence from systematic reviews of environmental influences on childhood overweight to prioritise areas for development of public health interventions.

Design Systematic literature reviews of intervention and longitudinal studies of environmental factors which could influence overweight via diet and/or physical activity, followed by a questionnaire-based ranking exercise for (a) the strength of the evidence for a causal association between the environmental factor and childhood overweight and (b) the likely effect size of public health actions on each factor on the prevalence of overweight in children. Environmental factors for which there was both strong evidence of causality and a larger effect size were selected for detailed modelling of the likely impact of public health interventions in children in Scotland.

Setting Children up to 8 years in Scotland.

Participants Academics and policy makers with experience of reviewing evidence of public health interventions on obesity.

Main Outcome Measure Ranking of environmental factors to identify those which should be prioritised for future public health interventions.

Results Of the twenty-four environmental factors initially identified at stakeholder workshops, no studies were found for three factors influencing physical activity and one factor affecting diet. For the remaining twenty factors a median of six (range 1-28) studies were identified and summarised in tabular form. Fourteen academics and policy makers were given the tables to complete the rating exercise, which took approximately $1 \mathrm{~h}$. The strength of the evidence and the likely effect size of actions were rated on a scale of 0 (low) to 5 (high). Eight of the environmental factors obtained mean ratings $>3$ for both strength of evidence and likely effect size: six were factors related to diet (high energy-dense snacks, sugar-sweetened soft drinks, infant feeding, availability of high fat, sugar and salt foods and portion size of manufactured foods and of restaurant and cafeteria items) and two were related to physical activity (physical education in schools and sedentary leisure activity). The ratings were lowest for access to local amenities, safe routes to schools and provision of healthy foods in schools.

Conclusion The ranking exercise provided a structured approach for obtaining a consensus view on priorities for public health action using tables of evidence from a systematic review. Use of this approach with a larger number academics and policy makers from different sectors would be useful to assess whether the area of expertise and sector (academia vs policy) influences the rating of the evidence.
L Spizzichino, D Galeone, L Pagliari, S Mele. Italian Ministry of Health, Prevention Department, Rome, Italy

Background In 2009, a health promotion initiative targeted the employees of the Italian Ministry Health aimed to promote compliance with the smoking ban and provide support for any smokers who wanted to quit, by providing free of charge tobacco cessation programme, available during working hours and in the workplace, for all smoking employees who agreed to participate in the programme. Activities included: an information seminar for employees on the risks of smoking; a questionnaire-based survey on the smoking habits of employees in the Ministry of Health headquarter; the launch of "Groups to quit smoking" run by personnel from the Italian anti-cancer league (LILT).

Results The programme is still ongoing but some results can be highlighted: The seminar held on February was attended by over 200 employees; all employees received an information booklet on the damage caused by smoking; the survey on the smoking habits of employees was completed. Out of the 1244 questionnaires distributed, 1030 were returned (participation rate $82.8 \%$ ), analyses were carried out on 994 of them. Data showed that $25.1 \%$ of employees are smokers, smoking an average of 13.3 cigarettes a day; $43 \%$ of them are heavy smokers. Sixty-three percent of smoking employees claim they want to quit, but only $40 \%$ of them were advised to do so by their physician. Non-smokers account for $50.5 \%$ of employees, and former smokers $24.4 \%$; second-hand smoke is a serious nuisance for $61 \%$ of them. Thirty-six percent of non-smokers is exposed to second-hand smoke in different places within the Ministry, including their own room and other rooms (in $2 \%$ and $6 \%$ of cases, respectively). Four tobacco cessation courses were launched in May 2009. A total of 74 employees (29\% of smoking employees) signed up. Of these, 57 participated, and after having completed the course, $32(56 \%)$ quit smoking and 25 (44\%) smoked fewer cigarettes a day. Follow-up activities 6 months after the completion of the course are currently ongoing, and the rate of abstention from smoking is above $37 \%$, in line with data reported in the literature. A new course has been launched in January 2010, 22 employees participated and at the end of the course $32 \%$ quit smoking.

Conclusions The program seems to work, law that bans smoking together with cessation program, free of charge and during working hours, are useful to protect non smokers from passive smoking and to help smokers to quit.

\section{P34 DEFINING A TARGET PROFILE FOR PROMOTING SMOKING CESSATION IN ITALY}

doi:10.1136/jech.2010.120477.34

${ }^{1} \mathrm{C}$ Bietta, ${ }^{2} \mathrm{G}$ Ferrante, ${ }^{2} \mathrm{~V}$ Minardi, ${ }^{3} \mathrm{~L}$ Spizzichino, ${ }^{3} \mathrm{~S}$ Vasselli. ${ }^{1}$ Local Health Unit, Region Umbria, Italy; ${ }^{2}$ Istituto Superiore di Sanità, National Centre of Epidemiology, Surveillance and Health Promotion, Rome, Italy; ${ }^{3}$ Prevention Department, Italian Ministry of Health, Rome, Italy

Background In Italy, smoking cessation programmes rarely consider the characteristics of people who make quit attempts during life (independently from quitting or still smoking) as an useful starting point for effective intervention. Behavioural risk factor surveillance systems can provide information about sociodemographic and health profile of smokers who express intention to quit smoking and therefore represent a potential target of cessation treatment and health promotion intervention.

Objective To evaluate the percentage of people who reported smoking cessation or at least an attempt once in the last 12 months; 
to investigate the association between the attempt and sociodemographic, behavioural and clinical variables.

Methods Data collected in 2008 from PASSI, the Italian behavioural risk factor surveillance system based on telephone interviews of residents aged $18-69$ years, were analysed to identify factors associated to quit smoking attempts.

Results Sample analysed included 18281 current and ex-smokers. Around two out of three people (11881) tried to quit smoking and among them 3911 (34\%) failed the attempt.

A multivariate analysis showed that smoking cessation attempt was more likely among males, elder (50-69 years) and married people; among those living with children ( $<14 \mathrm{yrs}$ ), with a temporary job, without economic difficulties and with a high level of education; among those with cardiovascular risk factors (hypertension, hypercholesterolaemia, obesity) and those affected by chronic diseases (almost one among cancer, diabetes and myocardial infarction). On the other side, sedentary people and those with good perceived health status are less intentioned to quit.

Stratification by sex showed a different strength of association between the outcome variable and other variables in males and females, but most important that education and perceived health status are not associated with the attempt to quit smoking among women.

Conclusions Population-based surveillance system tracking the characteristics of ex and current smokers, who tried to quit, may offer a lot of information, otherwise not available, for planning, implementing and evaluating promotion and prevention interventions to support people who try to quit smoking.

\section{P35 OBJECTIVE AND SUBJECTIVE METHOD OF PHYSICAL ACTIVITY MEASUREMENT IN A CROSS-SECTION OF ENGLISH ADULTS: HEALTH SURVEY FOR ENGLAND 2008}

doi:10.1136/jech.2010.120477.35

M Chaudhury, E Stamatakis, M Roth, J Mindell. Department of Epidemiology and Public Health, UCL, London, UK

Objective To describe and compare physical activity levels, sedentary and moderate-to-vigorous physical activity (MVPA) behaviour in HSE2008 in a representative sample of the adult population, using both objective (accelerometry) and subjective (self-report via questionnaire) methods of measurement.

Design Nationally representative cross-sectional population data from Health Survey for England, 2008.

Setting Random sample of the general population living in private households in England

Participants Subsample of 4507 adults aged $16+$ were selected for accelerometry wear, of whom 2115 adults had valid accelerometry data wear (with at least $600 \mathrm{~min}$ per day).

Main Outcome Measure Prevalence of those adults who meet the current physical activity (PA) recommendations by accelerometry data. Mean sedentary (minutes) and MVPA minutes (accelerometry).

Results Based on accelerometry data, $6 \%$ of men and $4 \%$ of women met the Chief Medical Officer's current minimum recommendations for PA by achieving at least 30 min of moderate or vigorous activity on at least 5 days in the week of accelerometer wear. Men and women aged 16-34 were most likely to have met the recommendations ( $11 \%$ and $8 \%$, respectively). In contrast, based on self-report measures, $39 \%$ of men and $29 \%$ of women were said to have met the PA recommendations. Only $10 \%$ of men and $8 \%$ of women whose self-reported activity level corresponded with meeting the recommendations also met the recommendations based on accelerometry. Overall, men had significantly longer periods of sedentary time per day than women (595 min and $584 \mathrm{~min}$, respectively), ( $p=0.003)$. While men spent an average of $31 \mathrm{~min}$ in MVPA in total per day, and women an average of 24 min, most of this was sporadic activity. Those who were not overweight or obese spent few minutes on average in sedentary time $(591 \mathrm{~min}$ for men and $577 \mathrm{~min}$ for women) than those who were obese (612 $\mathrm{min}$ for men and 585 for women). Similarly, adults not overweight or obese spent more MVPA minutes than those who were overweight or obese. This pattern was similar with each BMI category.

Conclusion Subjective self-reported method of assessing physical activity resulted in higher levels of activity than objective accelerometry data. Despite this, the results from objective accelerometer data corroborate self-report findings across age and sex. Objective measures provide more accurate data and should be used where available. Comparison between objective and subjective methods indicates that people over estimate their actual physical activity levels.

\section{P36 DISTRIBUTION OF PHYSICAL ACTIVITY AMENITIES IN SCOTLAND BY SMALL AREA MEASURES OF DEPRIVATION AND URBANICITY}

doi:10.1136/jech.2010.120477.36

${ }^{1} \mathrm{~K}$ E Lamb, ${ }^{1} \mathrm{~A}$ Ellaway, ${ }^{2} \mathrm{~N}$ S Ferguson, ${ }^{2} \mathrm{Y}$ Wang, ${ }^{3} \mathrm{D}$ Ogilvie. ${ }^{1} \mathrm{M}$ RC Social and Public Health Sciences Unit, Glasgow, UK; ${ }^{2}$ Department of Civil Engineering, University of Strathclyde, Glasgow, UK; ${ }^{3}$ MRC Epidemiology Unit and Centre for Diet and Activity Research (CEDAR), Cambridge, UK

Objective Obesity and physical activity are associated with aspects of the physical environment where people live, but the precise mechanisms of these associations are not well understood. Knowledge of the extent to which access to opportunities for physical activity is socially patterned is important to inform policy. A few studies of cities and regions (mostly conducted outside the UK) have found fewer resources in deprived areas, while others have not. The aim of this study was to examine the distribution of physical activity facilities by area level deprivation in Scotland, adjusting for differences in urbanicity and population density and specifically examining differences between Scotland's four largest cities.

Design A SportScotland data set, containing information about all Scottish sports facilities ( $\mathrm{n}=10283)$, was linked to Scottish Neighbourhood Statistics data on population size, deprivation category and urban classification for Scottish data zones. Poisson and negative binomial multilevel models were used to examine the association between facilities and deprivation quintiles, adjusting for random variation between local authorities.

Setting Scotland.

Results Prior to adjustment for urbanicity, the density of all facilities fell with increasing deprivation from quintiles 2 to 5 . After adjustment for urbanicity and local authority, the effect of deprivation remained significant but the pattern altered, with data zones in quintile 3 having the highest estimated mean density of facilities (2.63/1000 residents). The lowest estimated densities were observed in the most affluent $(1.99 / 1000)$ and the most deprived $(1.87 / 1000)$ quintiles, with no significant difference between these quintiles. After adjusting for population size, Dundee and Edinburgh had the highest estimated density of facilities whilst Glasgow had the lowest.

Conclusions Facility presence is dependent upon area level deprivation, after taking into account differences between local authorities and urban classification, with both the least and most deprived areas showing the lowest rates of facilities. The distribution of facilities across Scotland does appear to be socially patterned, but not according to a straightforward social gradient. There is a need to go further and take transport accessibility into account. 